\title{
A modified parametric iteration method for solving nonlinear second order BVPs
}

\author{
ASGHAR GHORBANI, MORTEZA GACHPAZAN* \\ and JAFAR SABERI-NADJAFI \\ Department of Applied Mathematics, School of Mathematical Sciences, \\ Ferdowsi University of Mashhad, Mashhad, Iran \\ E-mails: as_gh56@yahoo.com / gachpazan@math.um.ac.ir
}

\begin{abstract}
The original parametric iteration method (PIM) provides the solution of a nonlinear second order boundary value problem (BVP) as a sequence of iterations. Since the successive iterations of the PIM may be very complex so that the resulting integrals in its iterative relation may not be performed analytically. Also, the implementation of the PIM generally leads to calculation of unneeded terms, which more time is consumed in repeated calculations for series solutions. In order to overcome these difficulties, in this paper, a useful improvement of the PIM is proposed. The implementation of the modified method is demonstrated by solving several nonlinear second order BVPs. The results reveal that the new developed method is a promising analytical tool to solve the nonlinear second order BVPs and more promising because it can further be applied easily to solve nonlinear higher order BVPs with highly accurate.
\end{abstract}

Mathematical subject classification: Primary: 34B15; Secondary: 41A10.

Key words: modified parametric iteration method, parametric iteration method, second-order boundary value problems, numerical method.

\section{Introduction}

In this paper, we investigate the approximate analytical solution of the nonlinear second order BVPs of the type (with this assumption that the problem has the

\#CAM-213/10. Received: 26/V/10. Accepted: 25/X/10.

*Corresponding author. 
unique solution on $[a, b])$

$$
\left\{\begin{array}{l}
u^{\prime \prime}=F\left(x, u, u^{\prime}\right), \\
u(a)=\alpha, \quad u(b)=\beta,
\end{array}\right.
$$

by a new easy-to-use algorithm proposed in this work, which is based on the parametric iteration method (PIM) $[1,5]$. Here $a, b, \alpha$ and $\beta$ are the real constants and $F$ is a nonlinear continuous operator with respect to its arguments. These BVPs arise in engineering, applied mathematics and several branches of physics, and have attracted much attention. However, it is difficult to obtain closed-form solutions for BVPs, especially for nonlinear problems. In most cases, only approximate solutions (either numerical solutions or analytical solutions) can be expected. Some numerical methods such as finite difference method [4], finite element method [2] and shooting method [8] have been developed for obtaining approximate solutions to BVPs.

Recently, much attention has been focused on the analytic or numeric-analytic study of BVPs (e.g., see [6,9] and the references cited therein). Unlike the discrete solutions obtained by the purely numerical methods like the shooting methods, approximate analytical solutions can increase our insights into the natural behavior of complex systems. Analytical techniques do offer some options in acquiring solutions but they most habitually require some sort of linearization techniques for a successful effort. For those reasons, approximate analytical methods have been a foundation of study. One of the main advantages of the approximate analytical methods is its ability in providing us a continuous representation of the approximate solution, which allows better information of the solution over the time interval. On the other hand, the purely numerical methods provide solutions in discretized form, only at two ends of the time interval, thereby making it complicated in achieving a continuous representation.

Therefore, the strategy that will be pursued in this work rests mainly on establishing an effective and simple approximate analytical algorithm, requiring no tedious computational works, based on the PIM for solving the nonlinear second order BVPs. To demonstrate the utility of the modified method, in this study, some examples are given and we solve them using the new modified method and compare the obtained results with the numerical results. In all cases, the present technique performed excellently, as will shown later. 


\section{The basic idea of the PIM}

In this section, we describe the PIM for solving the general second-order BVP of (1). Then the local convergence is discussed.

\subsection{The PIM}

The PIM provides the solution of Eq. (1) as a sequence of approximations. The method gives rapidly convergent successive approximations of the exact solution if such a solution exists, otherwise approximations can be used for numerical purposes. The idea of the PIM is very simple and straightforward. Let $X=C^{2}[a, b]$, and $\mathcal{L}$ and $\mathcal{N}$ be the linear and nonlinear operators on $X$, respectively. To explain the basic idea of the PIM, we first consider Eq. (1) as below:

$$
\mathcal{L}[u(x)]+\mathcal{N}[u(x)]=f(x),
$$

where $\mathcal{L}$ with the property $\mathcal{L}[g] \equiv 0$ when $g \equiv 0$ denotes the so-called auxiliary linear operator with respect to $u, \mathcal{N}$ is a nonlinear continuous operator with respect to $u$ and $f(x)$ is the source term (here $u$ belongs to the intersection of domains $\mathcal{L}$ and $\mathcal{N}$, i.e. $u \in X$ ). Then we construct a family of iterative processes for Eq. (2) as [1, 5]:

$$
\mathcal{L}\left[u_{n+1}(x)-u_{n}(x)\right]=h H(x) \mathcal{A}\left[u_{n}(x)\right],
$$

with the boundary conditions

$$
u_{n+1}(a)=\alpha \quad \text { and } \quad u_{n+1}(b)=\beta,
$$

where

$$
\begin{aligned}
\mathcal{A}\left[u_{n}(x)\right] & =\mathcal{L}\left[u_{n}(x)\right]+\mathcal{N}\left[u_{n}(x)\right]-f(x) \\
& \equiv u_{n}^{\prime \prime}(x)-F\left(x, u_{n}(x), u_{n}^{\prime}(x)\right),
\end{aligned}
$$

and $u_{0}(x)$ is the initial guess (which can be freely chosen with possible unknown constants, or it can also be solved from its corresponding linear homogeneous equation $\mathcal{L}\left[u_{0}(x)\right]=0$ or linear nonhomogeneous equation $\left.\mathcal{L}\left[u_{0}(x)\right]=f(x)\right)$. 
The subscript $n$ denotes the $n$th iteration, $h \neq 0$ and $H(x) \neq 0$ denote the so-called auxiliary parameter and auxiliary function, respectively, which can be identified easily and efficiently by the techniques proposed in this paper. It should be emphasized that though we have the great freedom to choose the auxiliary linear operator $\mathcal{L}$, the auxiliary parameter $h$, the auxiliary function $H(x)$ and the initial approximation $u_{0}(x)$, which is fundamental to the validity and flexibility of the PIM, we can also assume that all of them are properly chosen so that solution of (3) exists, as will be shown in this paper later. Accordingly, the successive approximations $u_{n}(x) n \geq 0$ of PIM in the auxiliary parameter $h$ will be readily obtained by choosing the zeroth component. Consequently, the exact solution may be obtained by using

$$
u(x)=\lim _{n \rightarrow \infty} u_{n}(x) .
$$

Let $V=\left\{u: u \in C^{2}[a, b]\right\}$ be the solution space and $\left\{e_{k}(x) \mid e_{k}(x) \in\right.$ $V$ and $k=0,1,2, \cdots\}$ denote the set of base functions. Hence we can represent the solution in a series $u(x)=\sum_{k=0}^{\infty} c_{k} e_{k}(x)$ where $c_{k}$ is a coefficient. As long as a set of base functions is determined, the auxiliary linear operator $\mathcal{L}$, the initial approximation $u_{0}(x)$ and the auxiliary function $H(x)$ must be chosen in such a way that all solutions of the corresponding PIM equations (3) exist and can be expressed by this set of base functions. Now, in order to avoid expensive computational works for solving (1) via the PIM, it is straightforward to use the set of base functions

$$
\left\{(x-a)^{m} \mid m=0,1,2, \cdots\right\},
$$

to represent $u(x)$, i.e.,

$$
u(x)=\sum_{m=0}^{\infty} \gamma_{m}(x-a)^{m},
$$

where $\gamma_{m} \in \mathbb{R}(m=0,1,2, \cdots)$ are coefficients to be determined. In view of the solution expression (7) and according to the boundary conditions (4), it is straightforward to choose

$$
\mathcal{L}[u(x)]=u^{\prime \prime}(x),
$$

with the property (since we are dealing with the solution of the nonlinear second order BVPs, thus the property on $\mathcal{L}$ is determined from combining the first 
two terms of the set of base functions, that is, 1 and $(x-a))$

$$
\mathcal{L}\left[c_{1}(x-a)+c_{2}\right]=0,
$$

as the auxiliary linear operator where $c_{1}$ and $c_{2}$ are integral constants, and to choose an initial approximation of $u(x)$, which is the solution of the corresponding linear homogeneous equation $\mathcal{L}\left[u_{0}(x)\right]=0$,

$$
u_{0}(x)=\alpha+\frac{\alpha-\beta}{a-b}(x-a) .
$$

For simplicity, the auxiliary function $H(x)$ can be chosen in the form $H(x)=1$. According to (10), the solution of the PIM equation (3) becomes

$$
u_{n+1}(x)=u_{n}(x)+h \int_{a}^{x}(x-t) \mathcal{A}\left[u_{n}(t)\right] \mathrm{d} t+c_{1}(x-a)+c_{2},
$$

where $\mathcal{A}\left[u_{n}(t)\right]$ is defined as in (5) and the integral constants $c_{1}$ and $c_{2}$ are determined by imposing the boundary conditions (4).

We, therefore, obtain

$$
\begin{aligned}
u_{n+1}(x)= & u_{n}(x)+h \int_{a}^{x}(x-t) \mathcal{A}\left[u_{n}(t)\right] \mathrm{d} t \\
& -h \frac{x-a}{b-a} \int_{a}^{b}(b-t) \mathcal{A}\left[u_{n}(t)\right] \mathrm{d} t,
\end{aligned}
$$

where $\mathcal{A}\left[u_{n}(t)\right]=u_{n}^{\prime \prime}(t)-F\left(t, u_{n}(t), u_{n}^{\prime}(t)\right)$. Therefore, the successive approximations $u_{n}(x) \quad(n \geq 1)$ of the PIM iterative relationship of (13) in the auxiliary parameter $h$ will be readily obtained, especially by means of symbolic computation software such as Maple, Mathematica, Matlab and others.

\subsection{Convergence theorem}

The parametric iteration formula, (3), makes a recurrence sequence $\left\{u_{n}(x)\right\}$. Obviously, the limit of the sequence will be the solution of (1) if the sequence is convergent. In general cases, we can prove that, as long as the solution sequence (6) given by the PIM is convergent, it must be a solution of the problem (1). In the following, we give a new proof of convergence of the PIM, which details can be found in [5]. Here, $C^{2}[a, b]$ denotes the class of all real valued functions defined on $[a, b]$ which have continuous second-order derivative. 
Theorem 2.1. Let $F$ be a nonlinear continuous operator and for any positive integer $n, u_{n} \in C^{2}[a, b]$. Provided that the sequence (6) uniformly converges, where $u_{n}(x)$ is produced by the parametric iteration formulation of (3), it must be the exact solution of the problem (1).

Proof. If the sequence $u_{n}(x)$ converges, we can write

$$
U(x)=\lim _{n \rightarrow \infty} u_{n}(x)
$$

and it holds

$$
U(x)=\lim _{n \rightarrow \infty} u_{n+1}(x) .
$$

Using (14), (15) and the definition of $\mathcal{L}$, we can easily gain

$$
\lim _{n \rightarrow \infty} \mathcal{L}\left[u_{n+1}(x)-u_{n}(x)\right]=\mathcal{L} \lim _{n \rightarrow \infty}\left[u_{n+1}(x)-u_{n}(x)\right]=0 .
$$

From (16) and according to (3), we obtain

$$
\mathcal{L} \lim _{n \rightarrow \infty}\left[u_{n+1}(x)-u_{n}(x)\right]=h H(x) \lim _{n \rightarrow \infty} \mathcal{A}\left[u_{n}(x)\right]=0 .
$$

Since $h \neq 0$ and $H(x) \neq 0$, the relation (17) gives us

$$
\lim _{n \rightarrow \infty} \mathcal{A}\left[u_{n}(x)\right]=0 .
$$

From (18) and the continuity of $F$ operator, it holds

$$
\begin{aligned}
\lim _{n \rightarrow \infty} \mathcal{A}\left[u_{n}(x)\right] & =\lim _{n \rightarrow \infty}\left[u_{n}^{\prime \prime}(x)-F\left(x, u_{n}(x), u_{n}^{\prime}(x)\right)\right] \\
& =\lim _{n \rightarrow \infty} u_{n}^{\prime \prime}(x)-F\left(x, \lim _{n \rightarrow \infty} u_{n}(x), \lim _{n \rightarrow \infty} u_{n}^{\prime}(x)\right) \\
& =\left(\lim _{n \rightarrow \infty} u_{n}(x)\right)^{\prime \prime}-F\left(x, \lim _{n \rightarrow \infty} u_{n}(x),\left(\lim _{n \rightarrow \infty} u_{n}(x)\right)^{\prime}\right) \\
& =U^{\prime \prime}(x)-F\left(x, U(x), U^{\prime}(x)\right) .
\end{aligned}
$$

From the equations (18) and (19), we have

$$
U^{\prime \prime}(x)-F\left(x, U(x), U^{\prime}(x)\right)=0, \quad a \leq x \leq b .
$$


On the other hand, in view of (1), (4) and (15), it holds

$$
\begin{aligned}
& U(a)=\lim _{n \rightarrow \infty} u_{n+1}(a)=\alpha, \\
& U(b)=\lim _{n \rightarrow \infty} u_{n+1}(b)=\beta,
\end{aligned}
$$

Therefore, according to the above expressions, (20)-(22), $U(x)$ must be the exact solution of the problem (1) and this ends the proof.

It is clear that the convergence of the sequence (6) depends upon the initial guess $u_{0}(x)$, the auxiliary linear operator $\mathcal{L}$, the auxiliary parameter $h$ and the auxiliary function $H(x)$. Fortunately, the PIM provides us with great freedom of choosing them. Thus, as long as $u_{0}(x), \mathcal{L}, h$ and $H(x)$ are so properly chosen that the sequence (6) converges in a region $a \leq x \leq b$, it must converge to the exact solution in this region. Therefore, the combination of the convergence theorem and the freedom of the choice of above factors establishes the cornerstone of the validity and flexibility of the PIM.

\section{A modified PIM}

Since the successive iterations of the PIM may be very complex so that the resulting integrals in the relation (13) may not be performed analytically. Also, the implementation of the PIM generally leads to calculation of unneeded terms, which more time is consumed in repeated calculations for series solutions. In order to overcome these difficulties, in this section, a useful modification of the PIM is proposed. To completely eliminate these shortcomings in each step, provided that $\mathcal{A}\left[u_{n}(t)\right]$ in each of iterations is expanded in Taylor series around $a$, we suggest the following improvement of the PIM of (13), which is called the modified PIM (MPIM):

$$
u_{n+1}(x)=u_{n}(x)+h \int_{a}^{x}(x-t) G_{n}(t) \mathrm{d} t-h \frac{x-a}{b-a} \int_{a}^{b}(b-t) G_{n}(t) \mathrm{d} t,
$$

where

$$
\mathcal{A}\left[u_{n}(t)\right]=G_{n}(t)+O\left((t-a)^{n+1}\right) .
$$

It is noteworthy to point out that the MPIM formula (23) is capable of solving strongly nonlinear BVPs with the complicated variable coefficients in a straight- 
forward way. Furthermore, it can reduce the size of calculations. Most importantly, however, it is the fact that the MPIM algorithm (23) may solve a BVP exactly if its solution is an algebraic polynomial up to some degree.

\section{Choosing the auxiliary parameter $h$}

It is important to ensure that a solution series obtained using MPIM, which is always as a family of solution expressions in the auxiliary parameter $h$, is convergent in a large enough region whereby the convergence region and rate of solution series are dependent upon the auxiliary parameter $h$ and thus can be enlarged by choosing a proper value for $h$. Most important, however, it is how to choose the value of $h$ to make sure that the solution series converges fast enough in a large enough region. Since we have a family of solution expressions in the auxiliary parameter $h$, hence, regarding $h$ as an independent variable, a simple and practical way of selecting $h$ is to plot the curve of solution's derivatives with respect to in some points $[5,7]$. So, if the solution is unique, all of them converge to the same value and hence there exists a horizontal line segment in its figure that corresponds to a region of $h$ called the valid region of $h$. Thus, if we set $h$ any value in the so-called valid region of $h$, we are sure that the corresponding solution series converges. It is found that, for given initial approximation and the auxiliary function, the valid regions of are often nearly the same for a given problem. In most cases, we can find a proper value of $h$ to ensure that the solution series converges in the whole spatial/temporal regions. Therefore, the curves $h$ provide us with an easy way to show the influence of $h$ on the convergence region and rate of solution series.

\section{Results}

In this section, to give a clear overview of the content of this study, several BVPs will be tested by the proposed MPIM, which will ultimately show the simplicity, efficiency and accuracy of this method. All the results obtained here are calculated by using the symbolic calculus software Matlab 7. Moreover, the numerical method used in this paper to compare the numerical results is the Matlab bvp4c solver. 
Example 5.1. As the first example, we consider the following nonlinear second order BVP [3]:

$$
u^{\prime \prime}=\frac{1}{2} \exp \left(\frac{1}{2}(x+1) \cos (u-3 x+7)\right)
$$

subject to the boundary conditions

$$
u(-1)=-10 \quad \text { and } \quad u(1)=-4
$$

In order to solve the equation (25) using the MPIM algorithm, according to (2), we choose

$$
\begin{gathered}
X=C^{2}[-1,1], \\
\mathcal{L}[u(x)]=u^{\prime \prime}, \\
\mathcal{N}[u(x)]=-\frac{1}{2} \exp \left(\frac{1}{2}(x+1) \cos (u-3 x+7)\right)
\end{gathered}
$$

and $f(x) \equiv 0$ which $D(\mathcal{L})=D(\mathcal{N})=X$. So for this problem $u$ belongs to the intersection of these domains, i.e. $u \in X$.

Therefore, according to (23) and (24), we have the following MPIM iterative formula:

$$
u_{n+1}(x)=u_{n}(x)+h \int_{-1}^{x}(x-t) G_{n}(t) \mathrm{d} t-h \frac{x+1}{2} \int_{-1}^{1}(1-t) G_{n}(t) \mathrm{d} t,
$$

where

$$
u_{n}^{\prime \prime}(t)-\frac{1}{2} \exp \left(\frac{1}{2}(t+1) \cos \left(u_{n}(t)-3 t+7\right)\right)=G_{n}(t)+O\left((t+1)^{n+1}\right) .
$$

Starting with the initial approximation $u_{0}(x)=-10+3(x+1)$, we get

$$
\begin{aligned}
u_{1}(x)= & -10+\left(3+\frac{1}{2} h\right)(x+1)-\frac{1}{4} h(x+1)^{2}, \\
u_{2}(x)= & -10+\left(3+\frac{7}{6} h\right)(x+1)-\frac{1}{4}[h+h(1+h)] \\
& (x+1)^{2}-\frac{1}{24} h(x+1)^{3},
\end{aligned}
$$


To investigate the valid region $h$ of the solution obtained via the 10th-order MPIM, here we plot the curve of $u^{\prime}(-1)$ with respect to $h$, as shown in Figure 1 . According to this curve, it is easy to discover the valid region of $h$. We point out that the valid region of $h$ becomes more accurate as the number $n$ increases. It is usually convenient to investigate the valid region of $h$ of the MPIM by means of such kinds of the curves.

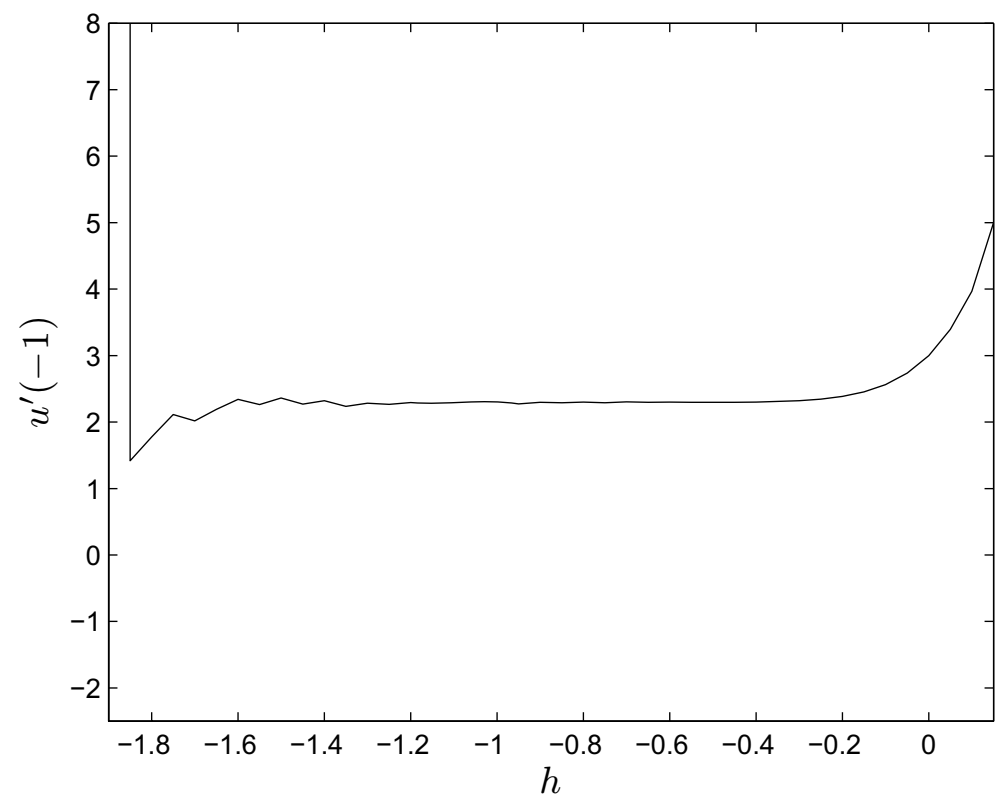

Figure 1 - The valid region of the auxiliary parameter $h$ using the 10th-order MPIM for Example 5.1.

Now, according to (23) and (24), by taking $n=9$, we can obtain a 10thorder MPIM approximation of (25) on [-1,1]. Figure 2 shows the approximate analytical solution obtained for Eq. (25) by using the 10th-order MPIM for $h=-0.5$ versus the numerical solution. It must be emphasized that our approximate solution applying the modified method is in excellent agreement with the numerical values.

Example 5.2. As the other example, we consider the following nonlinear second order BVP [3]:

$$
u^{\prime \prime}=\arctan (u)+2 u+\cos (x),
$$




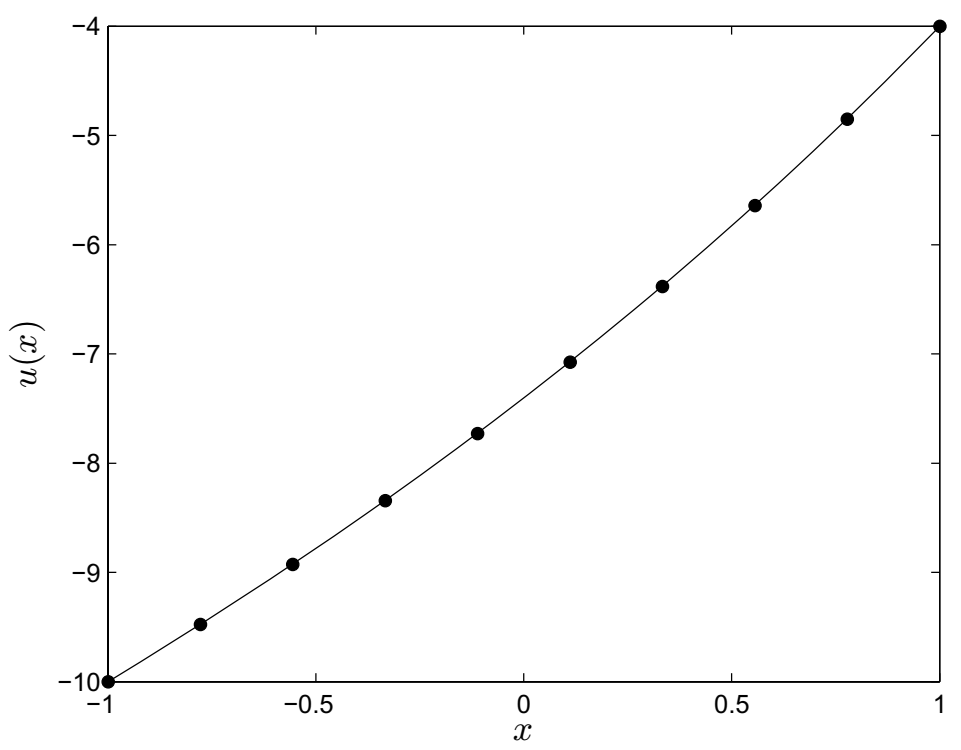

Figure 2 - Comparing the numerical solution (circle symbols) with the 10th-order MPIM solution when $h=-0.5$ (solid line) for Example 5.1.

subject to the boundary conditions

$$
u(0)=0 \quad \text { and } \quad u(1)=0 .
$$

In order to solve the equation (27) using the MPIM algorithm, according to (2), we choose $X=C^{2}[0,1], \mathcal{L}[u(x)]=u^{\prime \prime}, \mathcal{N}[u(x)]=-\arctan (u)-2 u$ and $f(x)=\cos (x)$ which $D(\mathcal{L})=D(\mathcal{N})=X$. So for this problem $u$ belongs to the intersection of these domains, i.e. $u \in X$.

The valid region $h$ and the approximate analytical solution of the 15th-order MPIM when $h=-0.4$ for Eq. (27) have been given in Figures 3 and 4, respectively.

Example 5.3. As the other example, we consider the following nonlinear second order BVP [9]:

$$
u^{\prime \prime}-s^{2} u-F s u^{2}+\frac{1}{M}=0,
$$

subject to the boundary conditions

$$
u(-1)=0 \quad \text { and } \quad u(1)=0 .
$$




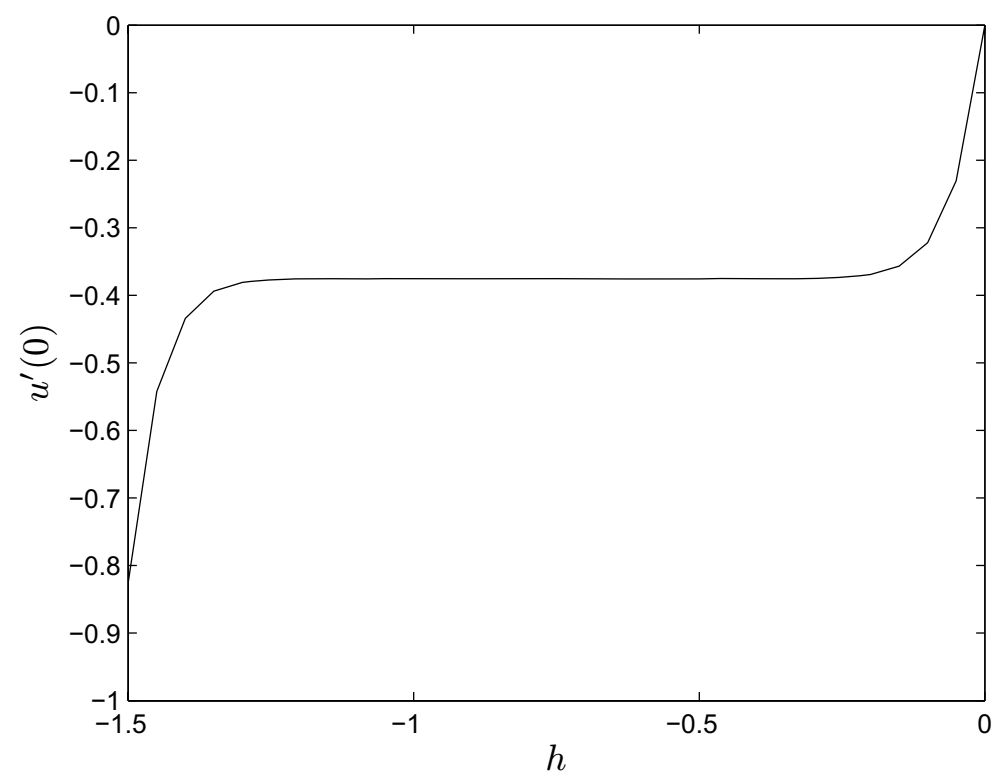

Figure 3 - The valid region of the auxiliary parameter $h$ using the 15th-order MPIM for Example 5.2.

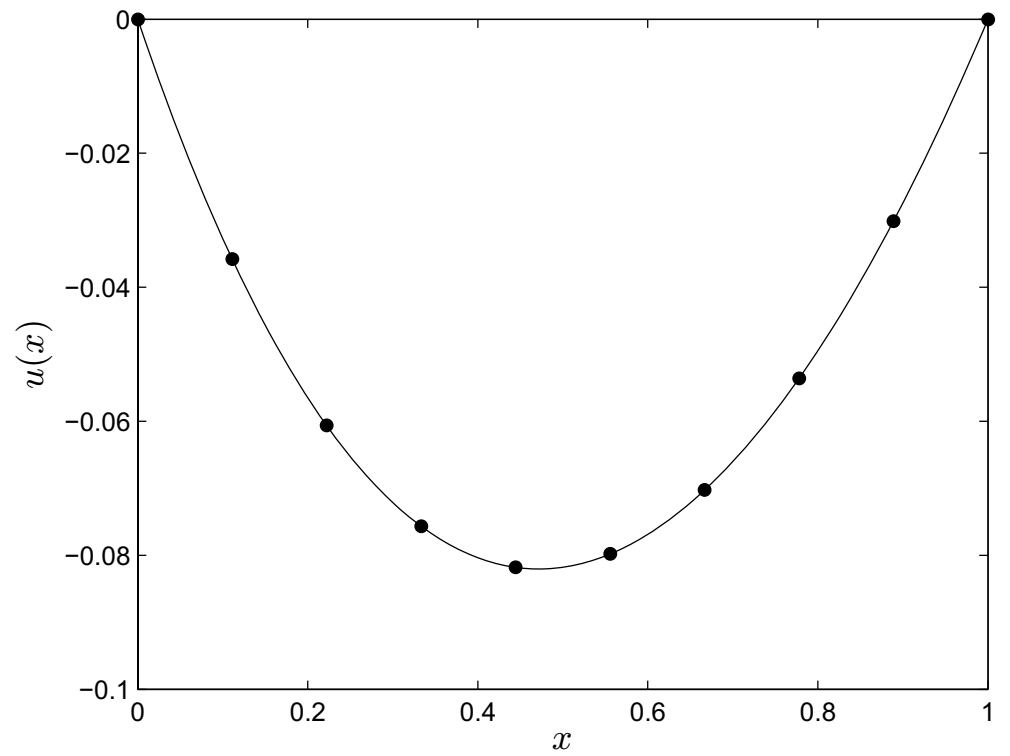

Figure 4 - Comparing the numerical solution (circle symbols) with the 15th-order MPIM solution when $h=-0.4$ (solid line) for Example 5.2. 
where the parameters are selected as in [9], that is, $s=F=M=1$.

In order to solve the equation (29) using the MPIM algorithm, according to (2), we choose $X=C^{2}[-1,1], \mathcal{L}[u(x)]=u^{\prime \prime}, \mathcal{N}[u(x)]=-s^{2} u-F s u^{2}$ and $f(x)=-\frac{1}{M}$ which $D(\mathcal{L})=D(\mathcal{N})=X$. So for this problem $u$ belongs to the intersection of these domains, i.e. $u \in X$.

The valid region $h$ and the approximate analytical solution of the 25th-order MPIM when $h=-0.25$ for Eq. (29) have been given in Figures 5 and 6, respectively.

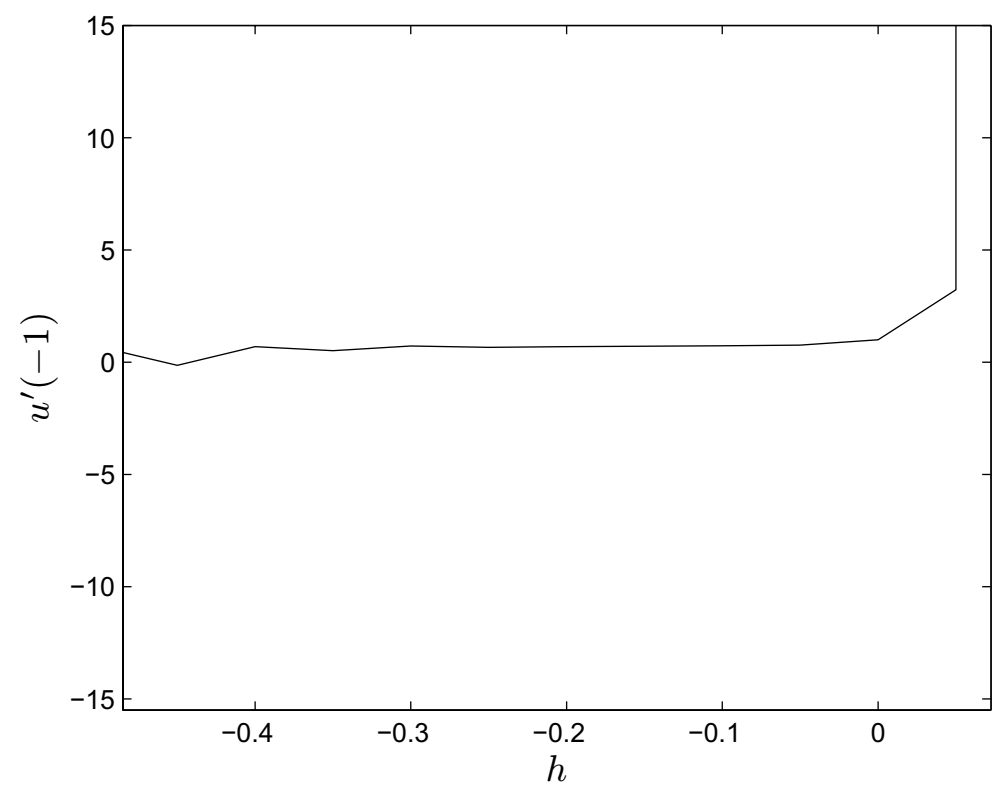

Figure 5 - The valid region of the auxiliary parameter $h$ using the 25th-order MPIM for Example 5.3.

It is interesting to point out that the MPIM algorithm proposed in this work is capable of solving the nonlinear second order BVPs of the general form $F\left(x, u, u^{\prime}, u^{\prime \prime}\right)=0$ subject to the boundary conditions $u(a)=\alpha$ and $u(b)=\beta$. For instance see the following example (Example 5.4).

Example 5.4. As the final example, we consider the following nonlinear second order BVP:

$$
u^{\prime \prime 5}+2 u u^{\prime \prime}-2 x u^{\prime}=32,
$$




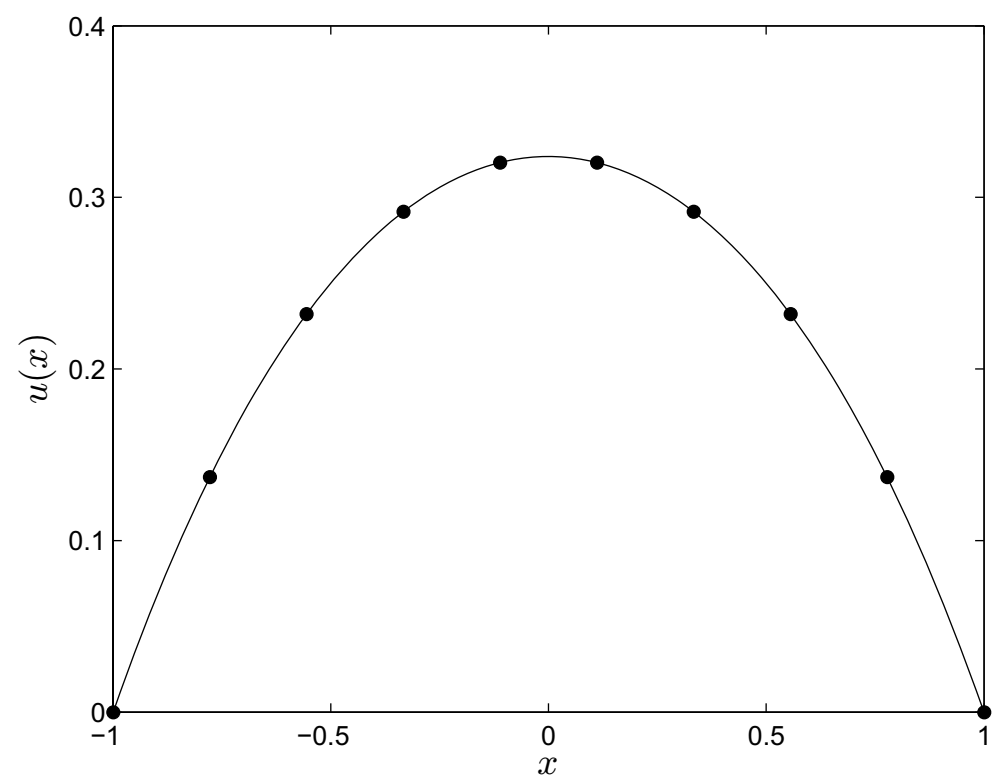

Figure 6 -Comparing the numerical solution (circle symbols) with the 25th-order MPIM solution when $h=-0.25$ (solid line) for Example 5.3.

subject to the boundary conditions

$$
u(-1)=1 \quad \text { and } \quad u(4)=16 .
$$

with the exact solution $u(x)=x^{2}$.

In order to solve the equation (31) using the MPIM algorithm, according to (2), we choose

$$
X=C^{2}[-1,4], \mathcal{L}[u(x)]=u^{\prime \prime}, \mathcal{N}[u(x)]=\sqrt[5]{32+2 x u^{\prime}-2 u u^{\prime \prime}}
$$

and $f(x) \equiv 0$ which $D(\mathcal{L})=D(\mathcal{N})=X$. So for this problem $u$ belongs to the intersection of these domains, i.e. $u \in X$.

The valid region $h$ and the approximate analytical solution of the 10th-order MPIM when $h=-1$ for Eq. (31) have been given in Figures 7 and 8, respectively.

In order to show the efficacy of the proposed MPIM, the results obtained using the MPIM for $u^{\prime}(a)$ and $u^{\prime}(b)$ are compared versus the numerical results in Tables 1 and 2. From the results Tables 1 and 2, it is easy to conclude that the MPIM is an effective analytic tool for solving the nonlinear second order BVPs. 


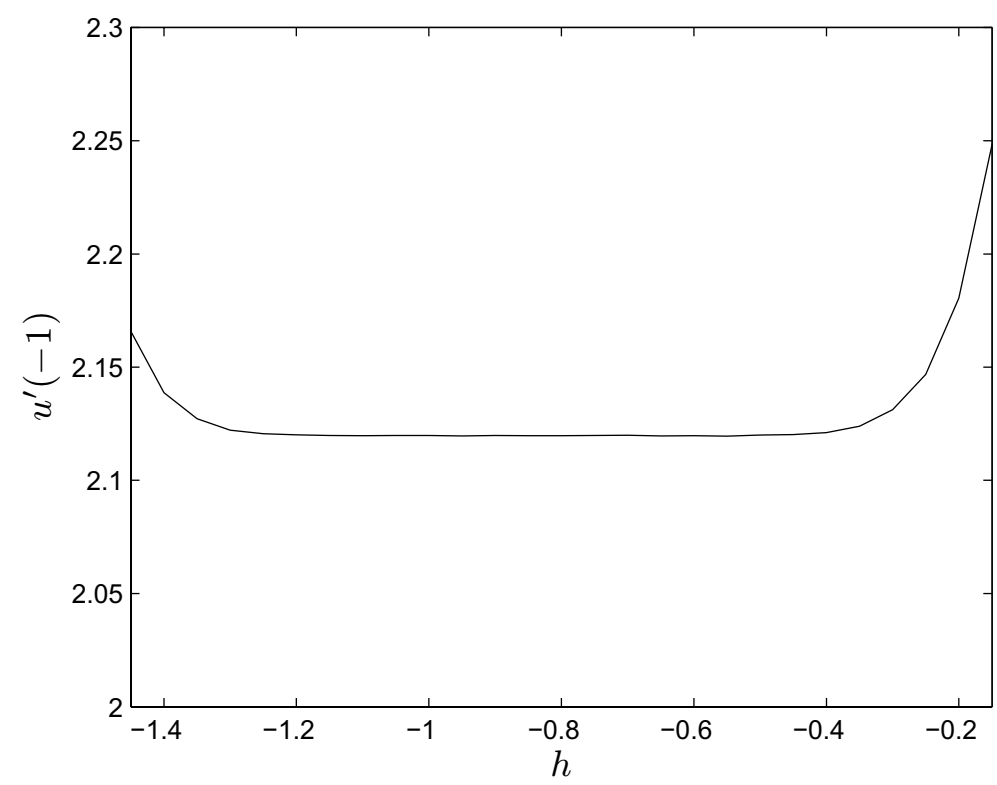

Figure 7 - The valid region of the auxiliary parameter $h$ using the 10th-order MPIM for Example 5.4.

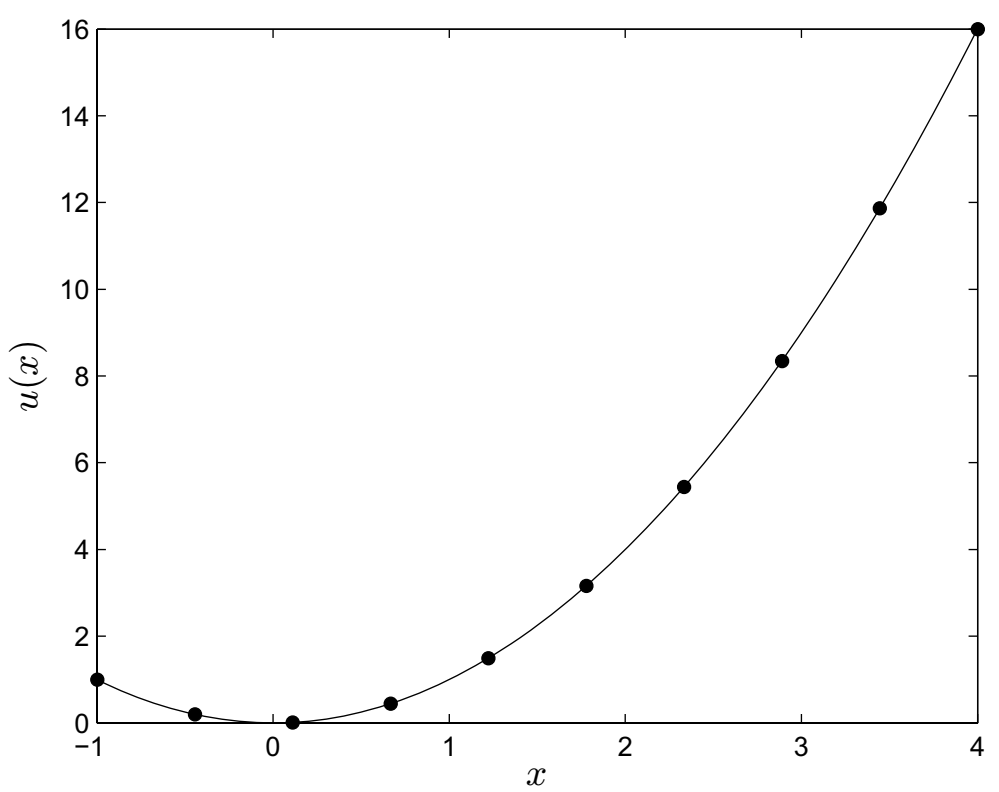

Figure 8 - Comparing the numerical solution (circle symbols) with the 10th-order MPIM solution when $h=-1$ (solid line) for Example 5.4. 


\begin{tabular}{c|c|c|c|c}
\hline Examples & Orders & $h$ & $u^{\prime}(a)$ of MPIM & $u^{\prime}(a)$ of numeric \\
\hline 5.1 & 10 & -0.50 & 2.2979739 & 2.2975254 \\
5.2 & 15 & -0.40 & -0.3755131 & -0.3755699 \\
5.3 & 25 & -0.25 & 0.7212678 & 0.7212354 \\
5.4 & 10 & -1.00 & -1.9999998 & -2.0000000 \\
\hline
\end{tabular}

Table 1 - Comparison of the values of the MPIM approximate solution for $u^{\prime}(a)$ with the numerical solution.

\begin{tabular}{c|c|c|c|c}
\hline Examples & Orders & $h$ & $u^{\prime}(b)$ of MPIM & $u^{\prime}(b)$ of numeric \\
\hline 5.1 & 10 & -0.50 & 3.9834993 & 3.9741175 \\
5.2 & 15 & -0.40 & 0.3011200 & 0.3013059 \\
5.3 & 25 & -0.25 & -0.721417 & -0.721235 \\
5.4 & 10 & -1.00 & 7.9999991 & 8.0000000 \\
\hline
\end{tabular}

Table 2 - Comparison of the values of the MPIM approximate solution for $u^{\prime}(b)$ with the numerical solution.

\section{Conclusions}

In this study we have proposed an effective modification of the parametric iteration method called the modified PIM (MPIM) to solve nonlinear second order boundary value problems. The obtained results demonstrate that the MPIM is easy to implement, accurate when applied to the nonlinear second order BVPs and avoid tedious computational works. Moreover, it can further be employed easily to solve nonlinear higher order BVPs with highly accurate.

Acknowledgments. The authors would like to express their sincere thanks to the reviewers for the careful reading of the manuscript and giving their valuable comments which helped to improve it considerably.

\section{REFERENCES}

[1] A. Ghorbani, Toward a new analytical method for solving nonlinear fractional differential equations. Comput. Meth. Appl. Mech. Engrg., 197 (2008), 4173-4179.

[2] A.G. Deacon and S. Osher, Finite-element method for a boundary-value problem of mixed type. SIAM J. Numer. Anal., 16 (1979), 756-778. 
[3] D.R. Kincaid and E.W. Cheny, Numerical Analysis Mathematics of Scientific Computing. California, Brooke Cole (2001).

[4] E. Doedel, Finite difference methods for nonlinear two-point boundary-value problems. SIAM J. Numer. Anal, 16 (1979), 173-185.

[5] J. Saberi-Nadjafi and A. Ghorbani, Piecewise-truncated parametric iteration method: a promising analytical method for solving Abel differential equations. Z. Naturforsch. 65a (2010), 529-539.

[6] S. Liang and D.J. Jeffrey, Approximate solutions to a parameterized sixth order boundary value problem. Comput. Math. Appl., 59 (2010), 247-253.

[7] Sh.J. Liao, Beyond perturbation: Introduction to the Homotopy Analysis Method. Boca Raton, Chapman \& Hall/CRC Press (2003).

[8] S.M. Roberts and J.S. Shipman, Two Point Boundary Value Problems: Shooting Methods. American Elsevier, New York (1972).

[9] S.S Motsa, P. Sibanda and S. Shateyi, A new spectral-homotopy analysis method for solving a nonlinear second order BVP. Commun. Nonlinear Sci. Numer. Simulat., 15 (2010), 2293-2302. 